\title{
Revisiting the J shaped curve, exploring the association between cardiovascular risk factors and concurrent depressive symptoms in patients with cardiometabolic disease: Findings from a large cross-sectional study
}

\author{
Bhautesh Dinesh Jani', Jonathan Cavanagh ${ }^{2}$, Sarah JE Barry ${ }^{3}$, Geoff Der ${ }^{4}$, Naveed Sattar ${ }^{5}$ and Frances S Mair ${ }^{6 *}$
}

\begin{abstract}
Background: Depression is common in patients with cardiometabolic diseases but little is known about the relationship, if any, between cardiovascular risk factor values and depressive symptoms in patients with these conditions. The objective of this paper is to study the association between cardiovascular risk factors and concurrent depressive symptoms in patients with three common cardiometabolic conditions: coronary heart disease $(\mathrm{CHD})$, stroke and diabetes.

Methods: We retrospectively reviewed primary care data for $\mathrm{N}=35537$ with 1 of the above 3 conditions who underwent depression screening using the depressive subscale of hospital anxiety and depression score (HADS-D). We reviewed 4 cardiometabolic risk factors (Systolic Blood Pressure [SBP], Diastolic Blood Pressure [DBP], BMI and total cholesterol) recorded concurrently in all patients and HbA1c in patients with diabetes $(n=18453)$. We analysed the association between individual risk factor value and a positive HADS-D screening result (>7) using logistic regression.

Results: SBP and BMI were noted to have a non-linear "J-shaped" relationship with the probability of having a positive HADS-D and observed nadirs (levels with the lowest probability) of $148 \mathrm{~mm} \mathrm{Hg}$ and $30.70 \mathrm{~kg} / \mathrm{m} 2$, respectively. Total cholesterol and DBP found to have a weaker curvilinear association with concurrent depression symptoms and nadirs of $3.60 \mathrm{mmol} / \mathrm{l}$ and $74 \mathrm{mmHg}$. Among patients with Diabetes, $\mathrm{HbA1c}$ was also found to have a "J-shaped" relationship with probability of having a positive HADS-D with an observed nadir of 7.06\% DCCT. The above relationships remain significant after adjusting for age, sex, socio-economic status and number of co-morbid conditions.

Conclusion: In patients with cardiometabolic disease, cardiovascular risk factor values at both extremes were associated with higher positive depression screening after adjusting for confounders. These findings have potentially important implications for clinical practice in relation to both risk stratification for depression and approaches to secondary prevention in individuals with cardiometabolic disease and merit further investigation to determine the nature and direction of the observed association.

Please see related article: http://www.biomedcentral.com/1741-7015/12/199.
\end{abstract}

Keywords: Cardiovascular risk factors, J-curve, Depression, Blood pressure, Body mass index, Total cholesterol, HbA1C, Diabetes, Stroke, Coronary heart disease

\footnotetext{
* Correspondence: frances.mair@glasgow.ac.uk

${ }^{6}$ Professor of Primary Care Research, General Practice and Primary Care, Institute of Health and Wellbeing, College of Medical, Veterinary and Life Sciences, University of Glasgow, Glasgow G112 9LX, UK

Full list of author information is available at the end of the article
} 


\section{Background}

Patients with chronic disease are two to three times more likely to suffer from depression when compared to the general population $[1,2]$. It is estimated that depression prevalence is $15-25 \%$ in patients with cardio-metabolic diseases such as coronary heart disease (CHD), diabetes and stroke [3-5]. Those with cardiometabolic disease who have suffered from depression have been reported to experience increased adverse clinical outcomes and mortality, and poorer functional abilities [4,6-8].

In 2008, the American Heart Association Science Advisory recommended routine depression screening for all patients with CHD [9]. However, there is no evidence to date that routine depression screening for patients with cardiometabolic disease leads to any improvement in depression or cardiac outcomes [10,11]. Moreover, there is some evidence in the UK and US to suggest that routine depression screening for all patients with cardiometabolic disease may struggle to achieve universal coverage [12-14]. In the UK, NICE(National Institute for Health and Care Excellence) recommends that depression screening or 'case finding' in patients with chronic disease should be targeted towards those who are believed to be 'high risk' [15]; but further research is needed to define who is at 'high risk'.

The relationship between depression and traditional cardiometabolic disease risk factors such as obesity, hypertension, hyperlipidaemia and raised HbA1c have been studied extensively in the general population. Depression is noted to have a significant positive association with obesity in the general population, with a stronger association noted in females [16,17]. In addition, evidence from longitudinal studies show that depression may have a bidirectional relationship with obesity [18]. Results from a meta-analysis of prospective cohort studies shows that depression increases the risk of hypertension incidence in the community [19]. A contradictory relationship has been observed between depression and hyperlipidaemia in elderly men and women in the community; with increased prevalence of depressive symptoms observed with low levels of high density lipoprotein cholesterol (higher atherogenic risk) in women and with low levels of low density lipoprotein cholesterol (lower atherogenic risk) in men [20]. In a prospective study of older adults in the general population, the probability of depression increased with raised HbA1c [21]. However, most of the evidence in this area has come from general population studies and there is a paucity of research in those with known cardiometabolic diseases who are likely to be subjected to treatment to reduce these risk factors.

Little is known about the relationship between cardiovascular risk factors and depressive symptoms in those with cardiometabolic disease. The aim of this project is to address this gap by studying the relationship, if any, between a range of cardiovascular risk factors (specifically SBP, DBP, total cholesterol, HbA1c and BMI) and depressive symptoms in those with three cardiometabolic conditions, namely, stroke, diabetes and CHD.

\section{Methods}

\section{Ethics statement}

We received approval from the West of Scotland research ethics committee to undertake this work. The work involved retrospective analysis of a large routinely collected dataset which was completely annonymised and the research team did not have access to patient identifiers, hence individual patient consent was not obtained. NHS Greater Glasgow and Clyde Enhanced Services data group, which was the authorised "guardian" of this data set, granted the permission to analyse the data.

\section{Study design and setting}

The data reported in this paper comes from the West of Scotland, with a population of circa 1.8 million served by two different health boards. The local health boards oversee a programme of incentivised depression screening in chronic disease as part of a wider chronic disease management programme of 'Local Enhanced Services' (LES). These are contractual arrangements at a local health board level with family practices where incentivisation is offered to primary care practitioners on certain indicators of chronic disease management. However, there are no penalties for non-adherence. In the areas under investigation in our study, family practices were paid under the LES scheme to carry out a comprehensive annual health assessment, which included depression screening, for patients with three common cardiometabolic conditions, CHD, diabetes and stroke. The annual health assessment was usually carried out by a practice nurse and lasted approximately one hour. The protocol for health assessment was specific for each of the three diseases but included monitoring of blood pressure (BP), total cholesterol, body mass index (BMI) and in those with diabetes, HbA1c. The assessment included detailed history taking, various physical examinations and blood tests.

\section{Participants}

We restricted our analysis to adults aged from 18 to 90 and health assessments recorded between 01/04/2008 to $31 / 03 / 2009$. A total of 125,143 patients were listed as having CHD, diabetes or stroke in the year 2008-09, the "DepChron" dataset [14], described in a previous publication. Of the total sample, 10,670 (8.5\%) patients were under treatment for depression and were thus exempt from screening. The remaining 114,473 (91.5\% of total sample size) patients were eligible for depression screening. However, the uptake of depression screening was 
poor and only undertaken in 35,537 (31.1\% of those eligible) and 78,936 (68.9\%) were not screened.

Measurement of clinical risk factors and outcome variable Systolic blood pressure (SBP) and diastolic blood pressure (DBP) measurements were recorded in $\mathrm{mm} \mathrm{Hg}$ and BMI in $\mathrm{kg} / \mathrm{m}^{2}$ determined from height and weight measurements. A blood sample was collected by the practice nurse at the time of assessment; the result for total cholesterol was reported in $\mathrm{mmol} / \mathrm{l}$ and $\mathrm{HbA} 1 \mathrm{c}$ was reported in Diabetes Control and Complications Trial (DCCT) units.

We restricted the values for cardiovascular risk factors to a clinically plausible range based on both our clinical judgement and the findings of general population studies. SBP measurements were restricted to a range between 90 to $240 \mathrm{~mm} \mathrm{Hg}$ and DBP to a range between 50 to $130 \mathrm{~mm} \mathrm{Hg}[22,23]$. Similarly, BMI was restricted to a range between 15 to55 [24], total cholesterol to 2-10 [25] and HbA1C to 3-18\% [26]. Observations in the data which were outside these range were excluded from the analysis. The depression subscale of HADS (HADS-D) gives a total score of 0 to 21 , and a threshold of $>7$ was used to define the presence of depressive symptoms, as endorsed by national guidelines [27]. The area based Scottish Index of Multiple Deprivations (SIMD) was used as a measure of socioeconomic status [28].

\section{Statistical analysis}

We used multiple logistic regression with the outcome variable as the prevalence of a positive screening for depression (defined as HADS-D >7). We used five separate regression models to examine the impact of each individual cardiovascular risk factor (SBP, DBP, total cholesterol, BMI, HbA1c) on the odds of a raised HADS-D. We entered quadratic terms for each clinical measure into regression models to allow for a non-linear relationship. We entered age (18-64 vs. 65-90), sex (male vs. female) and socio-economic status (deprived: SIMD deciles $1-5$ vs. affluent: SIMD deciles 6-10) into all of the models as binary variables. We also included the number of comorbid conditions (range 1-3, representing a combination of one or more of the three cardiometabolic disease under investigations: CHD, stroke or diabetes) into all regression models as a categorical variable. We present the results as a graph of the predicted probability of a raised HADS-D against corresponding values of the clinical risk factor. We calculated the turning point for each risk factor using the formula $\min =-\mathrm{b} / 2 \mathrm{a}$ where "a" represents coefficient of quadratic term and "b" represents coefficient of linear term.

We used the $R$ statistical software, version 3.0.2 for statistical analysis [29].

\section{Supplementary and sensitivity analyses}

The screened population was a subset of the whole dataset and the majority of the patients eligible for depression screening did not have HADS-D recorded due to poor uptake of depression screening. We compared the demographic features and distribution of clinical risk factors in both the screened population and the total population. We tested for interactions of each clinical risk factor with age, gender, number of comorbid condition and deprivation status for each of the corresponding regression models to check for potential effect modification. We also tested for cubic terms in each of the five regression models for five clinical measures. Sensitivity of the results to excluded values was assessed by repeating the analyses with all available patients.

We also performed multiple linear regression analysis with HADS-D as a continuous scale. We used five separate regression models to examine the impact of each individual cardiovascular risk factor (SBP, DBP, total cholesterol, BMI, HbA1c) on HADS-D as a continuous scale after excluding extreme values for each clinical measure as defined above. Quadratic and cubic terms for each clinical measure and other predictor variables, such as age, sex, socio-economic status and number of co-morbid conditions were added to the linear regression model as described above. The turning point or the "nadir" was calculated using the same formula described in the preceding section.

\section{Results}

\section{Sample size and characteristics}

$\mathrm{N}=35,537$ (32.5\% of total population) patients with one of the three chronic cardiometabolic diseases CHD, previous stroke and diabetes had results of depression screening with HADS-D recorded (see Figure 1). The demographic characteristics and cardiovascular risk factor distribution between the screened and total population were similar (please see "Additional file 1-Additional Analysis"). The HADS-D was positive ( $>7$ ) for 7080 patients (19.9\%). The demographic characteristics of the study sample are described in Table 1.

The distributions of the five cardiovascular risk factors in the study sample such as sample mean, standard deviation, missing values and the observations outside the plausible range considered and observed nadirs are described in Table 2.

\section{Blood pressure, total cholesterol, body mass index and depression}

SBP was found to have a "J-shaped" relationship with the probability of having a positive result with HADS-D screening, based on a regression model using all of the screened population with at least one of the three chronic diseases. The nadir or the minimum level of SBP with the 


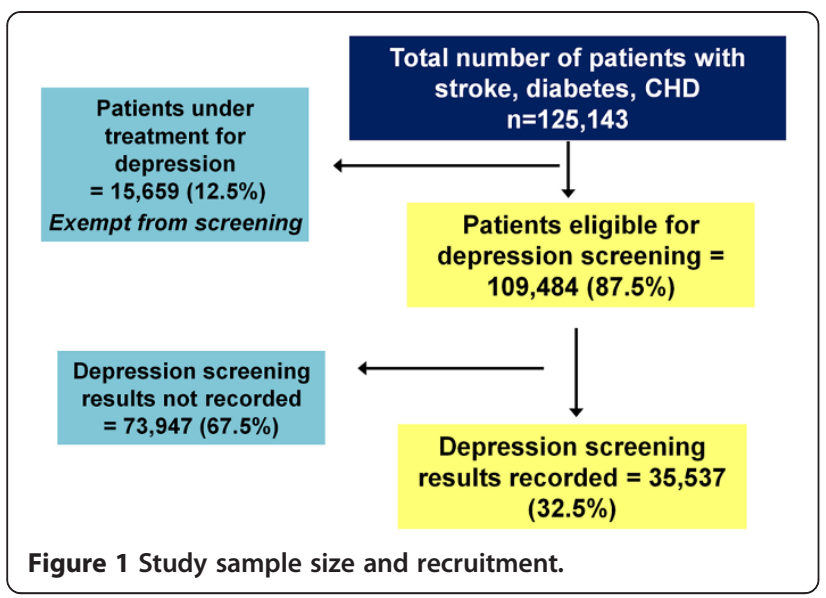

least probability of having a positive screening result with HADS-D was found to be $148 \mathrm{~mm} \mathrm{Hg}$ (see Figure 2). DBP was found to have a "J-shaped" relationship with the probability of having a positive result with HADS-D screening, based on a regression model using all of the screened population with at least one of the three chronic diseases. However, the shape of the J-curve was shallow for DBP when compared with SBP. The nadir for DBP with the least probability of having a positive screening result with HADS-D was found to be $74 \mathrm{~mm} \mathrm{Hg}$. This observed relationship between SBP, DBP and depressive symptoms remained significant after adjusting for age, sex, number of comorbid conditions and socio-economic status.

BMI was found to have a non-linear relationship with the probability of having a positive result with HADS-D screening, based on a regression model using all of the screened population with at least one of the three cardiometabolic conditions. The nadir or the minimum level for BMI was found to be $30.70 \mathrm{~kg} / \mathrm{m}^{2}$ (see Figure 2). Total Cholesterol was found to have a non-linear relationship

Table 1 Patient demographics of the study sample

\begin{tabular}{lll}
\hline & Demographics & $\begin{array}{l}\text { DepChron } \\
\text { (n=35,537) }\end{array}$ \\
\hline Age group & Missing & 11 \\
& $\mathbf{1 8 - 6 4}$ & $11553(32.52 \%)$ \\
& $\mathbf{6 4 - 9 0}$ & $23973(67.48 \%)$ \\
Gender & Missing & 18 \\
& Male & $20658(58.16 \%)$ \\
Deprivation status & Female & $14861(41.84 \%)$ \\
& Missing & 732 \\
& Deprived & $22726(65.30 \%)$ \\
Number of comorbid condition & Affluent & $12079(34.70 \%)$ \\
& Missing & 0 \\
& One & $27356(76.99 \%)$ \\
& Two & $7410(20.85 \%)$ \\
& Three & $771(2.16 \%)$ \\
\hline
\end{tabular}

with the probability of having a positive result with HADS-D screening (HADS-D > 7), based on the same regression model described above. However, the shape of the curve was less pronounced with lower values of total cholesterol and wider confidence intervals when compared to SBP and BMI. The nadir or the minimum level for total cholesterol was found to be $3.60 \mathrm{mmol} / \mathrm{l}$ (see Figure 2). The relationship between BMI and total cholesterol with probability of having HADS-D positive remained significant after adjusting for age, sex, number of comorbid conditions and socio-economic status.

\section{$\mathrm{HbA1C}$ and depression}

HbA1c was found to have a non-linear "J-shaped" relationship with the probability of having a positive result with HADS-D screening (HADS-D > 7), based on a regression model using only patients with diabetes $(\mathrm{n}=18,453$, missing $=2775$, excluded $=2)$. The shape of the curve was more similar but the confidence intervals were slightly wider, when compared to SBP, DBP and BMI. The nadir or the minimum level for HbA1c was found to $7.06 \%$ DCCT ( $54 \mathrm{mmol} / \mathrm{mol}$ IFCC) (see Figure 3). This relationship also remained significant after adjusting for age, sex, number of comorbid conditions and socio-economic status.

\section{Supplementary and sensitivity analysis}

There were no significant cubic terms for any of the cardiovascular risk factors. There were significant interactions between DBP and sex $(\mathrm{p}$-value $=0.01)$ and BMI and age $(p$-value $=0.009)$ (please see "Additional file 1Additional Analysis"). Hence, we calculated the nadirs separately for these groups with significant interactions. The nadirs for DBP were $78 \mathrm{~mm} \mathrm{Hg}$ for males and $63 \mathrm{~mm} \mathrm{Hg}$ for females respectively. The nadirs for BMI were $32.12 \mathrm{~kg} / \mathrm{m} 2$ for those aged18-64 years and $29.54 \mathrm{~kg} / \mathrm{m} 2$ for those $65-90$ years respectively. The shape of the curve was unchanged for DBP and BMI after doing sub-group analysis for sex and age respectively (please see "Additional file 1-Additional Analysis"). The results were unchanged after including extremes of clinical values outside the clinically plausible range described above (please see "Additional file 1-Additional Analysis").

The five cardiovascular risk factors had a non-linear relationship in the respective linear regression models, after adjusting for age, sex, socio-economic status and number of co-morbid conditions. The observed nadirs for SBP, DBP, total cholesterol and BMI were $145 \mathrm{~mm}$ $\mathrm{Hg}, 78 \mathrm{~mm} \mathrm{Hg}, 3.41 \mathrm{mmol} / \mathrm{l}$ and $30.25 \mathrm{~kg} / \mathrm{m} 2$ respectively. The observed nadir for $\mathrm{HbA1c}$ in patients with diabetes was $6.21 \mathrm{DCCT}(44.4 \mathrm{mmol} / \mathrm{mol}$ IFCC). The value for HADS-D increased with increase in value of these clinical measures above their respective nadirs but 
Table 2 Study sample $(n=35537)$ distribution for the five clinical risk factors, missing values, the number of extreme observations outside the usually observed clinically plausible values, analyzed data and observed nadirs

\begin{tabular}{|c|c|c|c|c|c|}
\hline Clinical measure (range included) & Mean (SD) & $\mathrm{N}$ missing & Exclusions & $\mathrm{N}$ analyzed & Observed nadirs \\
\hline \multirow[t]{2}{*}{ Systolic BP (90-240) } & $133(17.54)$ & 3398 & $\mathrm{n}<90=110$ & 32029 & $148 \mathrm{~mm} \mathrm{Hg}$ \\
\hline & & & $n>240=0$ & & \\
\hline \multirow[t]{2}{*}{ Diastolic BP (50-130) } & $74 \cdot 57(10.32)$ & 3398 & $\mathrm{n}<50=165$ & 31972 & $74 \mathrm{~mm} \mathrm{Hg}$ \\
\hline & & & $n>130=2$ & & \\
\hline \multirow[t]{2}{*}{ Body mass index (15-55) } & $28 \cdot 95(6.02)$ & 5398 & $\mathrm{n}<15=29$ & 30042 & $30.70 \mathrm{~kg} / \mathrm{m} 2$ \\
\hline & & & $n>55=68$ & & \\
\hline \multirow[t]{2}{*}{ Total cholesterol $(2-10)$} & $4 \cdot 31(1.05)$ & 4226 & $\mathrm{n}<2=50$ & 31244 & $3.60 \mathrm{mmol} / \mathrm{l}$ \\
\hline & & & $n>10=17$ & & \\
\hline \multirow[t]{2}{*}{$\mathrm{HbA1c}(3-18)$} & $7 \cdot 52(1.68)$ & 2775 & $\mathrm{n}<3=2$ & 15676 & $7.06 \mathrm{DCCT}$ \\
\hline & & & $n>18=0$ & & \\
\hline
\end{tabular}

Legend: $\mathrm{BP}=$ Blood Pressure; $\mathrm{n}=18453$ for HbA1c.

it increased with decrease in value below these observed nadirs. There were no significant cubic terms. The results of each linear regression are presented in detail in "Additional file 2- Linear Regression with HADS-D as continuous measure".

\section{Discussion}

In a large, community based sample of patients with $\mathrm{CHD}$, previous stroke, or diabetes depressive symptoms assessed using depression screening were found to have a nonlinear association with five routine cardiovascular risk factors of disease management. The relationships were 'J-shaped' with high levels of SBP and BMI associated with greater levels of concurrent depressive symptoms, but with the lowest levels also associated with increased prevalence of depressive symptoms. DBP and total Cholesterol had a similar but weaker relationship with depression. In patients with diabetes, a "J-shaped" relationship was again observed between HbA1c levels and depressive symptoms. These associations remained significant after adjusting for demographic factors such as age, sex, number of comorbid conditions and socioeconomic status; including or excluding clinical observations with extreme values and using HADS-D as continuous scale.

Previous evidence studying the relationship between cardiovascular risk factor values and depressive symptoms has mainly come from general population studies. Barrett-Connor et al. reported a non-linear relationship between DBP and depression with a observed nadir of $75 \mathrm{~mm} \mathrm{Hg}$ DBP for concurrent depressive symptoms in a general population sample [30]. In various cross-sectional studies involving mainly elderly population, depression has been observed to have a non-linear association with SBP [31-34] and DBP [30,35]. Similarly, increased prevalence of depressive symptoms has been observed with extreme values of total cholesterol [36,37] and HbA1c in general population samples [38], in a non-linear trend. There is as yet no published literature that we know of that examines the relationship between cardiovascular risk factors and depressive symptoms in those with cardiometabolic disease.
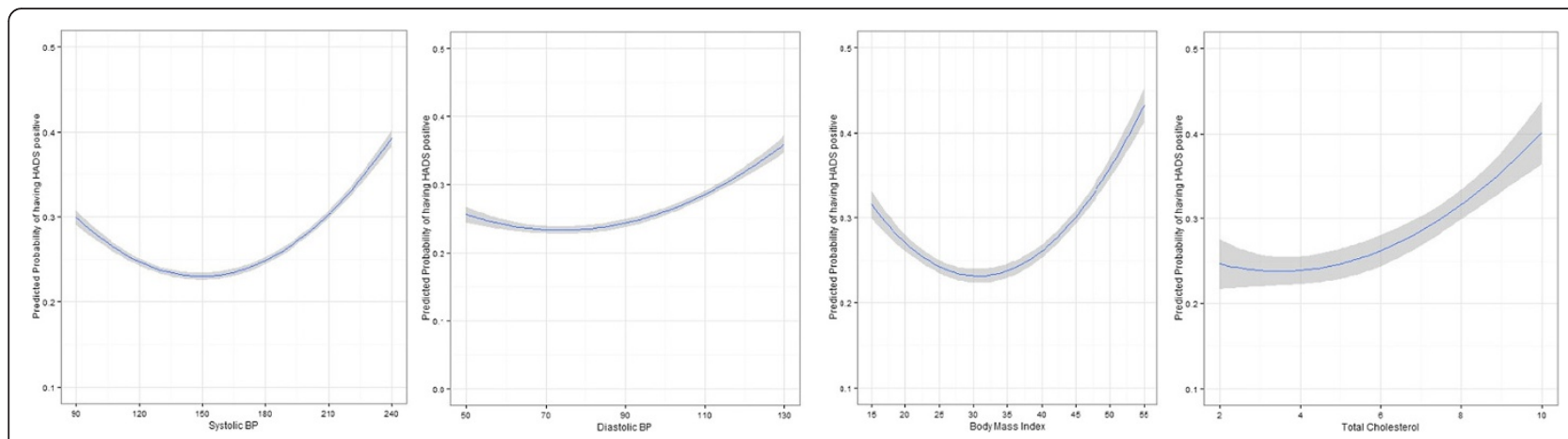

Figure 2 Relationship of Systolic BP (SBP), Diastolic BP (DBP), Body Mass Index (BMI) and total cholesterol with probability of having a positive HADS-D (>7) with $95 \%$ confidence intervals. 


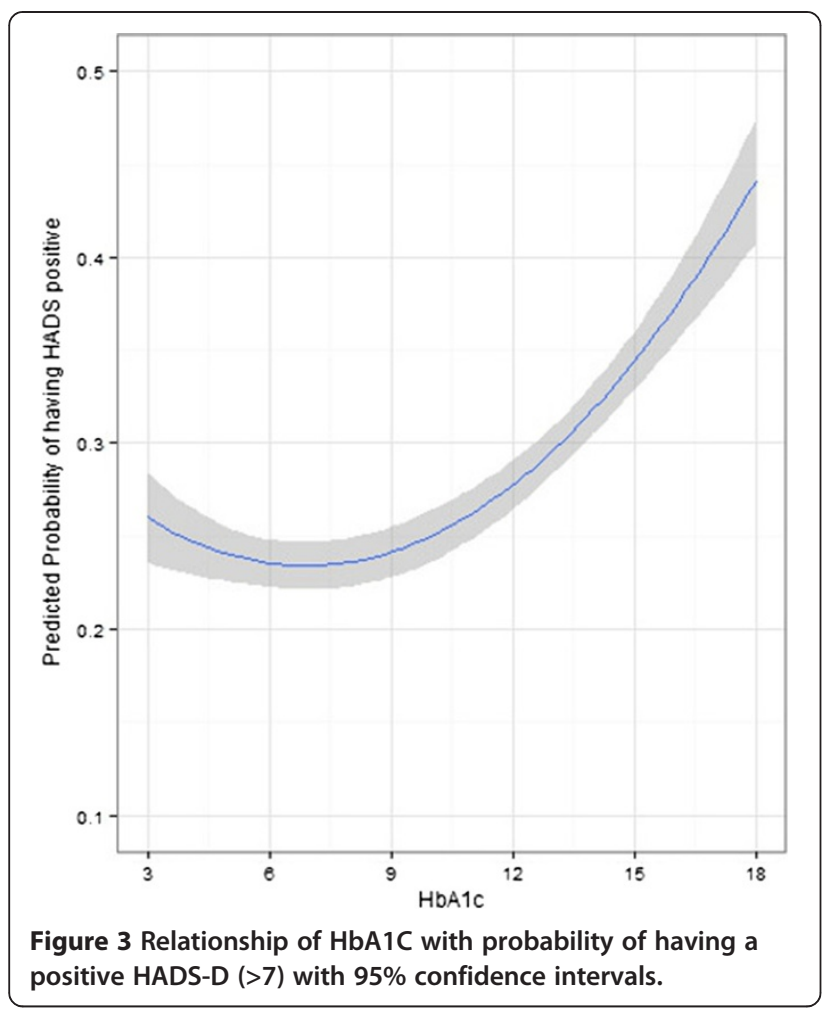

Non-linear relationship between extreme values of SBP, DBP, BMI and HbA1c and adverse clinical outcomes such as increased incidence of vascular events and deaths in patients with cardiometabolic conditions has been reported extensively [39-43].

There are two potential implications of our findings. Firstly, if the association between extreme values of these risk factors with depressive symptoms in those with cardiometabolic disease is supported by prospective studies, then this relationship could be used to identify those at "high risk" of depression. This would then offer a mechanism for targeting of depression screening in those with cardiometabolic disease. Secondly, these results need to be replicated using other datasets and also prospectively to further explain the nature and direction of the observed association between depressive symptoms and cardiovascular risk factors values. Such further investigation is necessary in order to determine whether the lower cardiovascular risk factors are merely markers of other disease processes (for example, low total cholesterol levels associated with malnutrition, liver diseases and haematological diseases) [44-46] that may make patients more vulnerable to experiencing depressive symptoms or whether it could be attributed to a potential side-effect of aggressive cardiovascular risk factor management [47-50].

This study has a number of key strengths. The data came from a large, community based sample, and importantly reflecting real life clinical practice. There are several limitations. As the study was based on cross-sectional analysis, it is not possible to make causal inferences from the findings of this study. It is therefore unclear whether the observed non-linear association of cardiovascular risk factors with prevalent depressive symptoms is due to cause or effect.

Secondly, we did not have complete information on biobehavioural factors such as smoking status, alcohol intake and levels of physical activity which are likely to influence the values of cardiovascular risk factors considered and also the prevalence of depressive symptoms [51-54].

Since only a minority of the patients were actually screened, depression status was unknown for a large number of patients, which remains an important limitation. There may be important differences between patients with known depression status and those whose depression status was unknown, which are not clearly evident from their baseline demographic data. Practitioners may intuitively screen those patients where they are more likely to get a positive result, for instance patients with multimorbidity. Also, there is a possibility of reverse causality with GPs reviewing a patient whom they consider to have depression and offering screening subsequently. Previously reported barriers to discussing depression (or mental health) in patients with chronic disease in primary care, such as stigma associated around the 'label' and physicians' preconception of normalizing depression in patients with chronic disease, could be influencing factors behind low uptake of depression screening in our study $[55,56]$.

Finally, the overall accuracy of depression screening in our study was reliant on HADS-D which is a self-reported measure and it is not a gold standard measure for assessing depressive symptoms in patients with cardiometabolic disease in a primary care setting $[11,57,58]$. We also did not have information on history of previous episodes of depression for patients in our study which may influence the prevalence levels for depressive symptoms.

\section{Conclusion}

In a general practice sample of patients with CHD, stroke, or diabetes, depressive symptoms were found to have a strong curvilinear association with SBP, BMI, and HbA1c; and a weaker curvilinear association with total cholesterol and DBP. Further investigation of these relationships is urgently needed to clarify the nature of these associations, in order to determine whether they have potentially important implications for clinical practice in relation to either risk stratification for depression or our approach to secondary prevention in individuals with cardiometabolic disease. 


\section{Additional files}

Additional file 1: "Additional Analysis". Results of sensitivity and supplementary analysi.

Additional file 2: "Linear Regression with HADS-D as continuous measure". Results of statistical analysis with linear regression models using HADS-D as a continuous measure.

\section{Competing interests}

The authors declared that they have no competing interests.

\section{Author contributions}

BDJ, JC, NS, SB and FSM designed the study and obtained the funding. BDJ, $\mathrm{SB}$ and $\mathrm{GD}$ analysed the data. All authors interpreted the findings and contributed to the drafting of this paper. FSM is the guarantor and the lead author for this manuscript. All authors read and approved the final manuscript.

\section{Acknowledgement}

The study was funded by Chief Scientist Office, Scotland CAF/12/04. The funders had no role in study design, data collection and analysis, decision to publish, or preparation of the manuscript.

\section{Author details}

${ }^{1}$ CSO Clinical Fellow, General Practice and Primary Care, Institute of Health and Wellbeing, College of Medical, Veterinary and Life Sciences, University of Glasgow, Glasgow G12 9LX, UK. ${ }^{2}$ Professor of Psychiatry, Mental Health and Wellbeing, Sackler Institute, Institute of Health and Wellbeing, College of Medical, Veterinary and Life Sciences, University of Glasgow, Neurology Block, Southern General Hospital, Glasgow G51 4TF, UK. ${ }^{3}$ Consultant Biostatistician, Robertson Centre for Biostatistics, Institute of Health and Well Being, College of Medical, Veterinary and Life Sciences, University of Glasgow, Glasgow G12 8QQ, UK. ${ }^{4}$ Statistician, MRC/CSO Social and Public Health Sciences Unit, Institute of Health and Wellbeing, College of Medical, Veterinary and Life Sciences, University of Glasgow, Glasgow G12 8RZ, UK. ${ }^{5}$ Professor of Metabolic Medicine, BHF Glasgow Cardiovascular Research Centre, Institute of Cardiovascular and Medical Sciences, College of Medical, Veterinary and Life Sciences, University of Glasgow, Glasgow G12 8TA, UK. ${ }^{6}$ Professor of Primary Care Research, General Practice and Primary Care, Institute of Health and Wellbeing, College of Medical, Veterinary and Life Sciences, University of Glasgow, Glasgow G112 9LX, UK.

Received: 24 July 2014 Accepted: 3 October 2014 Published: 28 October 2014

\section{References}

1. Egede LE: Major depression in individuals with chronic medical disorders: prevalence, correlates and association with health resource utilization, lost productivity and functional disability. Gen Hosp Psychiatry 2007, 29:409-416.

2. Moussavi S, Chatterji S, Verdes E, Tandon A, Patel V, Ustun B: Depression, chronic diseases, and decrements in health: results from the World Health Surveys. Lancet 2007, 370:851-858

3. Ali S, Stone MA, Peters JL, Davies MJ, Khunti K: The prevalence of co-morbid depression in adults with Type 2 diabetes: a systematic review and meta-analysis. Diabet Med 2006, 23:1165-1173.

4. Hadidi N, Treat-Jacobson DJ, Lindquist R: Poststroke depression and functional outcome: a critical review of literature. Hear Lung 2009, 38:151-162.

5. Whooley MA, De JP, Vittinghoff E, Otte C, Moos R, Carney RM, Ali S, Dowray S, Na B, Feldman MD, Schiller NB, Browner WS: Depressive symptoms, health behaviors, and risk of cardiovascular events in patients with coronary heart disease. JAMA 2008, 300:2379-2388.

6. Larsen KK, Christensen B, Sondergaard J, Vestergaard M: Depressive Symptoms and Risk of New Cardiovascular Events or Death in Patients with Myocardial Infarction: A Population-Based Longitudinal Study Examining Health Behaviors and Health Care Interventions. PLoS One 2013, 8:e74393.

7. Katon WJ, Rutter C, Simon G, Lin EH, Ludman E, Ciechanowski P, Kinder L, Young B, Von KM: The association of comorbid depression with mortality in patients with type 2 diabetes. Diabetes Care 2005, 28:2668-2672.
8. Pozuelo L, Tesar G, Zhang J, Penn M, Franco K, Jiang W: Depression and heart disease: what do we know, and where are we headed? Cleve Clin J Med 2009, 76:59-70.

9. Lichtman JH, Bigger JT, Blumenthal JA, Frasure-Smith N, Kaufmann PG, Lespérance F, Mark DB, Sheps DS, Taylor CB, Froelicher ES: Depression and coronary heart disease: recommendations for screening, referral, and treatment. Circulation 2008, 118:1768-1775.

10. Ontario HQ: Screening and management of depression for adults with chronic diseases: an evidence-based analysis. Ont Health Technol Assess Ser 2013, 13:1-45.

11. Thombs BD, Roseman M, Coyne JC, de Jonge P, Delisle VC, Arthurs E, Levis B, Ziegelstein RC: Does Evidence Support the American Heart Association's Recommendation to Screen Patients for Depression in Cardiovascular Care? An Updated Systematic Review. PLoS One 2013, 8:e52654.

12. Smolderen KG, Buchanan DM, Amin AA, Gosch K, Nugent K, Riggs L, Seavey $G$, Spertus JA: Real-world lessons from the implementation of a depression screening protocol in acute myocardial infarction patients: implications for the American Heart Association depression screening advisory. Circ Qual Outcomes 2011, 4:283-292.

13. Burton C, Simpson C, Anderson N: Diagnosis and treatment of depression following routine screening in patients with coronary heart disease or diabetes: a database cohort study. Psychol Med 2013, 43:529-537.

14. Jani BD, Purves D, Barry S, Cavanagh J, McLean G, Mair FS: Challenges and Implications of Routine Depression Screening for Depression in Chronic Disease and Multimorbidity: A Cross Sectional Study. PLoS One 2013, 8:e74610.

15. NICE: Depression in Adults with a Chronic Physical Health Problem: Treatment and Management; 2009.

16. Scott KM, Bruffaerts R, Simon GE, Alonso J, Angermeyer M, de Girolamo G, Demyttenaere K, Gasquet I, Haro JM, Karam E, Kessler RC, Levinson D, Medina Mora ME, Oakley Browne MA, Ormel J, Villa JP, Uda H, Von Korff M: Obesity and mental disorders in the general population: results from the world mental health surveys. Int J Obes (Lond) 2008, 32:192-200.

17. De Wit L, Luppino F, van Straten A, Penninx B, Zitman F, Cuijpers P: Depression and obesity: A meta-analysis of community-based studies. Psychiatry Res 2010, 178:230-235.

18. Luppino FS, de Wit LM, Bouvy PF, Stijnen T, Cuijpers P, Penninx BWJH, Zitman FG: Overweight, obesity, and depression: a systematic review and meta-analysis of longitudinal studies. Arch Gen Psychiatry 2010, 67:220-229.

19. Meng $L$, Chen D, Yang $Y$, Zheng $Y$, Hui R: Depression increases the risk of hypertension incidence: a meta-analysis of prospective cohort studies. J Hypertens 2012, 30:842-851.

20. Ancelin ML, Carrire I, Boulenger JP, Malafosse A, Stewart R, Cristol JP, Ritchie K, Chaudieu I, Dupuy AM: Gender and genotype modulation of the association between lipid levels and depressive symptomatology in community-dwelling elderly (The ESPRIT Study). Biol Psychiatry 2010, 68:125-132.

21. Hamer M, Batty GD, Kivimaki M: Haemoglobin A1c, fasting glucose and future risk of elevated depressive symptoms over 2 years of follow-up in the English Longitudinal Study of Ageing. Psychol Med 2011, 41:1889-1896.

22. Pater C: The Blood Pressure "Uncertainty Range" - a pragmatic approach to overcome current diagnostic uncertainties (II). Curr Control Trials Cardiovasc Med 2005, 6:5.

23. Wolf-Maier K, Cooper RS, Banegas JR, Giampaoli S, Hense H-W, Joffres M, Kastarinen M, Poulter N, Primatesta P, Rodríguez-Artalejo F, Stegmayr B, Thamm M, Tuomilehto J, Vanuzzo D, Vescio F: Hypertension prevalence and blood pressure levels in 6 European countries, Canada, and the United States. JAMA 2003, 289:2363-2369.

24. Penman $A D$, Johnson WD: The changing shape of the body mass index distribution curve in the population: implications for public health policy to reduce the prevalence of adult obesity. Prev Chronic Dis 2006, 3:A74.

25. Thompson R, O'Regan C, Morant S, Phillips B, Ong S: Measurement of baseline total cholesterol: new data from The Health Improvement Network (THIN) database. Prim Care CardiovasC J 2008, 1:107.

26. Currie CJ, Peters JR, Tynan A, Evans M, Heine RJ, Bracco OL, Zagar T, Poole CD: Survival as a function of $\mathrm{HbA}(1 \mathrm{c})$ in people with type 2 diabetes: a retrospective cohort study. Lancet 2010, 375:481-489.

27. NICE: Depression in Adults (update); 2009. 
28. Scottish Index of Multiple Deprivation (SIMD). In http://www.scotland.gov. uk/Topics/Statistics/SIMD.

29. Team RC: R Core Team (2012). R: A language and environment for statistical computing. R Foundation for Statistical Computing, Vienna, Austria. In 2012. ISBN 3-900051-07-0. http://www.R-project.org/.

30. Barrett-Connor E, Palinkas LA: Low blood pressure and depression in older men: a population based study. BMJ 1994, 308:446-449.

31. Stroup-Benham CA, Markides KS, Black SA, Goodwin JS: Relationship between low blood pressure and depressive symptomatology in older people. J Am Geriatr Soc 2000, 48:250-255.

32. Pilgrim JA, Stansfeld S, Marmot M: Low blood pressure, low mood? BMJ 1992, 304:75-78

33. Niu K, Hozawa A, Awata S, Guo H, Kuriyama S, Seki T, Ohmori-Matsuda K, Nakaya N, Ebihara S, Wang Y, Tsuji I, Nagatomi R: Home blood pressure is associated with depressive symptoms in an elderly population aged 70 years and over: a population-based, cross-sectional analysis. Hypertens Res 2008, 31:409-416.

34. Kim B-S, Bae JN, Cho MJ: Depressive symptoms in elderly adults with hypotension: different associations with positive and negative affect. $J$ Affect Disord 2010, 127:359-364.

35. Jorm AF: Association of hypotension with positive and negative affect and depressive symptoms in the elderly. Br J Psychiatry 2001, 178:553-555.

36. Morgan RE, Palinkas LA, Barrett-Connor EL, Wingard DL: Plasma cholesterol and depressive symptoms in older men. Lancet 1993, 341:75-79.

37. Steegmans PH, Hoes AW, Bak AA, van der Does E, Grobbee DE: Higher prevalence of depressive symptoms in middle-aged men with low serum cholesterol levels. Psychosom Med 2000, 62:205-211.

38. Kivimaki M, Tabak AG, Batty GD, Singh-Manoux A, Jokela M, Akbaraly TN, Witte DR, Brunner EJ, Marmot MG, Lawlor DA: Hyperglycemia, Type 2 Diabetes, and Depressive Symptoms: The British Whitehall II study. Diabetes Care 2009, 32:1867-1869.

39. Bangalore S, Qin J, Sloan S, Murphy SA, Cannon CP: What is the optimal blood pressure in patients after acute coronary syndromes?: Relationship of blood pressure and cardiovascular events in the PRavastatin OR atorVastatin Evaluation and Infection Therapy-Thrombolysis In Myocardial Infarction (PROVE IT-TI. Circulation 2010, 122:2142-2151.

40. Cushman WC, Evans GW, Byington RP, Goff DC, Grimm RH, Cutler JA, Simons-Morton DG, Basile JN, Corson MA, Probstfield JL, Katz L, Peterson KA, Friedewald WT, Buse JB, Bigger JT, Gerstein HC, Ismail-Beigi F: Effects of intensive blood-pressure control in type 2 diabetes mellitus. N Engl J Med 2010, 362:1575-1585.

41. Messerli FH, Panjrath GS: The J-curve between blood pressure and coronary artery disease or essential hypertension: exactly how essential? J Am Coll Cardiol 2009, 54:1827-1834.

42. Romero-Corral A, Montori VM, Somers VK, Korinek J, Thomas RJ, Allison TG, Mookadam F, Lopez-Jimenez F: Association of bodyweight with total mortality and with cardiovascular events in coronary artery disease: a systematic review of cohort studies. Lancet 2006, 368:666-678.

43. Gerstein HC, Miller ME, Byington RP, Goff DC, Bigger JT, Buse JB, Cushman WC, Genuth S, Ismail-Beigi F, Grimm RH, Probstfield JL, Simons-Morton DG, Friedewald WT: Effects of intensive glucose lowering in type 2 diabetes. N Engl J Med 2008, 358:2545-2559.

44. Ghadir MR, Riahin AA, Havaspour A, Nooranipour M, Habibinejad AA: The relationship between lipid profile and severity of liver damage in cirrhotic patients. Hepat Mon 2010, 10:285-288.

45. Marini A, Carulli G, Azzarà A, Grassi B, Ambrogi F: Serum cholesterol and triglycerides in hematological malignancies. Acta Haematol 1989, 81:75-79.

46. Kovesdy CP, Anderson JE, Kalantar-Zadeh K: Inverse association between lipid levels and mortality in men with chronic kidney disease who are not yet on dialysis: effects of case mix and the malnutritioninflammation-cachexia syndrome. J Am Soc Nephrol 2007, 18:304-311.

47. O'Neil A, Sanna L, Redlich C, Sanderson K, Jacka F, Williams L, Pasco JA, Berk M: The impact of statins on psychological wellbeing: a systematic review and meta-analysis. BMC Med 2012, 10:154.

48. Macedo AF, Taylor FC, Casas JP, Adler A, Prieto-Merino D, Ebrahim S: Unintended effects of statins from observational studies in the general population: systematic review and meta-analysis. BMC Med 2014, 12:51.

49. Zureik M, Courbon D, Ducimetière P: Serum cholesterol concentration and death from suicide in men: Paris prospective study I. BMJ 1996, 313:649-651.
50. Olson MB, Kelsey SF, Matthews KA, Bairey Merz CN, Eteiba W, McGorray SP, Cornell CE, Vido DA, Muldoon MF: Lipid-lowering medication use and aggression scores in women: a report from the NHLBI-sponsored WISE study. J Womens Health (Larchmt) 2008, 17:187-194.

51. Sullivan LE, Fiellin DA, O'Connor PG: The prevalence and impact of alcohol problems in major depression: a systematic review. Am J Med 2005, 118:330-341.

52. Van der Meer RM, Willemsen MC, Smit F, Cuijpers P: Smoking cessation interventions for smokers with current or past depression. Cochrane Database Syst Rev 2013, 8:CD006102.

53. Chaiton MO, Cohen JE, O'Loughlin J, Rehm J: A systematic review of longitudinal studies on the association between depression and smoking in adolescents. BMC Public Health 2009, 9:356.

54. Blumenthal JA, Babyak MA, Carney RM, Huber M, Saab PG, Burg MM, Sheps D, Powell L, Taylor CB, Kaufmann PG: Exercise, depression, and mortality after myocardial infarction in the ENRICHD trial. Med Sci Sports Exerc 2004, 36:746-755.

55. Karasz A, Dowrick C, Byng R, Buszewicz M, Ferri L, Olde Hartman TC, Van DS, van Weel-Baumgarten $\mathrm{E}$, Reeve J: What we talk about when we talk about depression: doctor-patient conversations and treatment decision outcomes. Br J Gen Pract 2012, 62:e55-e63.

56. Coventry PA, Hays R, Dickens C, Bundy C, Garrett C, Cherrington A, ChewGraham C: Talking about depression: a qualitative study of barriers to managing depression in people with long term conditions in primary care. BMC Fam Pract 2011, 12:10.

57. Haddad M, Walters P, Phillips R, Tsakok J, Williams P, Mann A, Tylee A: Detecting Depression in Patients with Coronary Heart Disease: $A$ Diagnostic Evaluation of the PHQ-9 and HADS-D in Primary Care, Findings From the UPBEAT-UK Study. PLoS One 2013, 8:e78493.

58. Meader N, Mitchell AJ, Chew-Graham C, Goldberg D, Rizzo M, Bird V, Kessler D, Packham J, Haddad M, Pilling S: Case identification of depression in patients with chronic physical health problems: a diagnostic accuracy meta-analysis of 113 studies. Br J Gen Pract 2011, 61:e808-e820.

doi:10.1186/1471-2261-14-139

Cite this article as: Jani et al.: Revisiting the J shaped curve, exploring the association between cardiovascular risk factors and concurrent depressive symptoms in patients with cardiometabolic disease: Findings from a large cross-sectional study. BMC Cardiovascular Disorders 2014 14:139.

\section{Submit your next manuscript to BioMed Central and take full advantage of:}

- Convenient online submission

- Thorough peer review

- No space constraints or color figure charges

- Immediate publication on acceptance

- Inclusion in PubMed, CAS, Scopus and Google Scholar

- Research which is freely available for redistribution
C) Biomed Central 